\title{
NEUROSCIENCE
}

\section{Mapping gene expression in C.elegans neurons}

Taylor, S.R. et al. Cell https://doi.org/10.1016/j.cell.2021.06.023 (2021)

Caenorhabditis elegans was the first model organism to have its connectome completed. The connectome, which traces all neurons and their synaptic connections, provides essential insight into the structure of the nervous system. However, inferring function from structure alone can be difficult and more research is needed to characterize the C. elegans nervous system and bridge the gap between circuit structure and function.

In a new study published in Cell, a team of investigators from Vanderbilt University, Columbia University and Yale University used Fluorescence Activated Cell Sorting (FACS) and single-cell RNA sequencing (scRNA-seq) to produce the gene expression profiles of all 302 neurons in the nervous system of the hermaphrodite C. elegans. "We expect that these datasets and the tools that we have developed for interrogating them will power future investigations into the genetic basis of neuronal connectivity and function," write the investigators in their report.
To ensure that they profiled the entire C. elegans nervous system, Taylor and colleagues isolated neurons at the L4 larval stage, when all neuron types have been generated and most neurons have terminally differentiated. They used a series of different fluorescent marker strains to isolate the different subsets of neurons within the nervous system. Sc-RNA-seq data analysis of 100,955 sequenced cells from 17 experiments identified 128 transcriptionally distinct neuron types (classes and subclasses) that expressed a distinct set of neuropeptide-encoding genes and neuropeptide receptors.

Further analysis of scRNA-seq results also revealed that different neuron types expressed different transcription factors and RNA-binding proteins, and identified over 200 cis-regulatory elements in the neuronal transcriptomes. These findings could inform future studies on gene regulation in the C. elegans nervous system.

"This catalog of gene expression provides an essential foundation for a comprehensive exploration of transcriptional and gene regulatory patterns that lead to neuronal diversity, connectivity, and function. C. elegans is the first organism in which a complete anatomical map of its nervous system is matched with a nervous system-wide molecular map, therefore providing new opportunities to investigate neuronal development and function," conclude the investigators.

Additional information can be found on the website of the C. elegans Neuronal Gene Expression Map \& Network (CeNGEN) consortium at https://cengen. org. The raw sequencing results are available on GEO (GSE136049). The investigators also developed a Web interface, CengenApp (https://cengen. shinyapps.io/CengenApp) to facilitate analysis of the dataset.

\section{Alexandra Le Bras}

Published online: 9 August 2021 https://doi.org/10.1038/s41684-021-00835-7

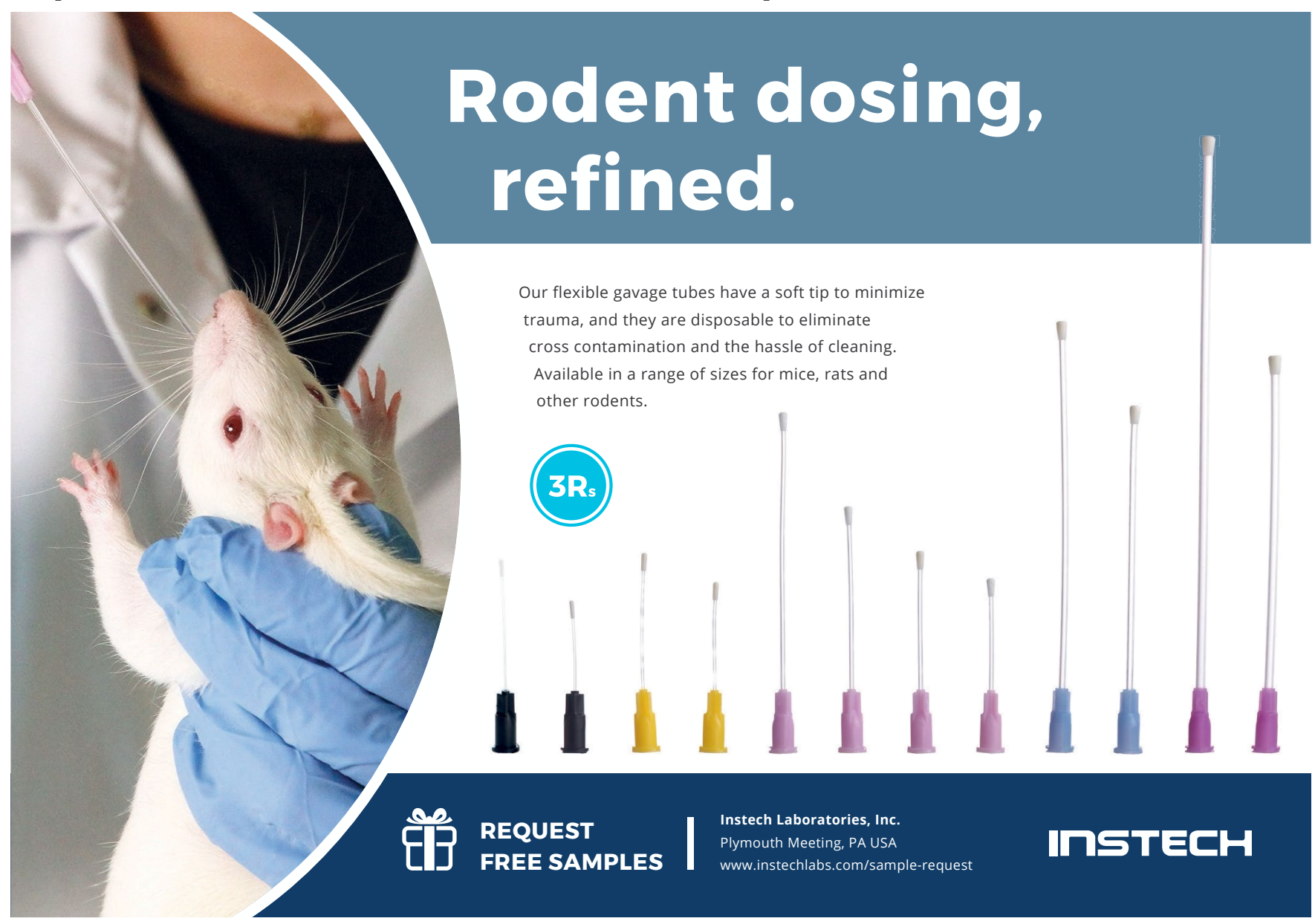

